\title{
Photonic Nanojets and Whispering Gallery Modes in Smooth and Corrugated Micro-Cylinders under Point-Source Illumination
}

\author{
Ibrahim Mahariq $^{1, *(\mathbb{D})}$, Thabet Abdeljawad ${ }^{2,3,4, * \mathbb{D}}$, Abdullah S. Karar ${ }^{1}$ (D), Shadi A. Alboon ${ }^{1,5}$, \\ Hamza Kurt ${ }^{6}$ (D) and Alexey V. Maslov ${ }^{7}$
}

1 College of Engineering and Technology, American University of the Middle East, Al-Eqaila 54200, Kuwait; abdullah.karar@aum.edu.kw (A.S.K.); shadi.alboon@aum.edu.kw (S.A.A.)

2 Department of Mathematics and General Sciences, Prince Sultan University, P. O. Box 66833, Riyadh 11586, Saudi Arabia

3 Department of Medical Research, China Medical University, Taichung 40402, Taiwan

4 Department of Computer Science and Information Engineering, Asia University, Taichung 41354, Taiwan

5 Electronics Engineering Department, Hijjawi Faculty for Engineering Technology, Yarmouk University, Irbid 21163, Jordan

6 Department of Electrical and Electronics Engineering, Faculty of Engineering, TOBB University of Economics and Technology, Ankara 06560, Turkey; hkurt@etu.edu.tr

7 Department of Radiophysics, University of Nizhny Novgorod, 603950 Nizhny Novgorod, Russia; avmaslov@rf.unn.ru

* Correspondence: ibrahim.maharik@aum.edu.kw (I.M.); tabdeljawad@psu.edu.sa (T.A.); Tel.: +965-2225-1400 (I.M.)

Received: 15 June 2020; Accepted: 15 July 2020; Published: 21 July 2020

\begin{abstract}
We numerically investigate the generation of photonic nanojets (PNJs) and the excitation of whispering gallery modes (WGMs) supported by both smooth and corrugated dielectric micro-cylinders under point-source illumination. Results show that the location of the point-source defines the location and properties of PNJs, whereas stability of WGMs exists in smooth micro-cylinders but vanishes in corrugated ones. It is shown that the location of the point-source acts as an additional degree of freedom for controlling the characteristics of the generated PNJs for both smooth and corrugated dielectric micro-cylinders. Furthermore, the influence of the point-source location on the stability of the excited WGMs was diminished for the smooth micro-cylinders, while being fully pronounced for their corrugated counterparts.
\end{abstract}

Keywords: photonic nanojets; point-source illumination; whispering gallery modes; dielectric micro-cylinders

\section{Introduction}

Photonic nanojets (PNJs) arise from the near-field scattering of light from dielectric structures on the micrometer scale [1-30]. The formed nanojet is specially localized with high intensity, while exhibiting a diameter smaller than the diffraction limit across a wide range of optical wavelengths. These unique properties offer a potentially novel near-field optical microscopy technique, which does not only overcomes the diffraction limitation of conventional optical microscopy, but also eliminates the reliance on evanescent fields [4,5]. Photonic nanojet have also been used in several applications, such as enhanced Raman scattering, biosensing, sub-diffraction-limited optical imaging, and single molecule detection [6-8]. In addition, photonic nanojets are shown to be attractive for high-density data storage, lithography, high-resolution microscopy, low-loss optical wave guides, and nonlinear optical 
effects $[9,10]$. There has been considerable research and development on the theoretical aspects [4], experimental demonstrations [5], and direct applications [11] of photonic nanojets in the literature.

Under specific illumination conditions photonic nanojets disappear and instead a whispering gallery mode (WGM) is excited, as reported in the literature [12,13,15-17]. In Ref. [13], the authors explore the optical resonance dynamics of dielectric micro-cylinders under plane-wave illumination. The associated on-resonance and off-resonance characteristics were numerically demonstrated by using both Mie theory and the spectral element method (SEM). Specifically, the authors demonstrate that slight detuning in the radius and refractive index of the dielectric cylinder deteriorate the resonance condition and create a photonic nanojet. In addition, the authors in [15], report that strong power enhancement up to 3 orders of magnitude is attainable with small micro-cylinders. As the refractive index changes from 1.50 to 2.25 , the corresponding required radius needed to support WGMs is captured through careful fine-tuning.

WGMs have not only been realized in smooth dielectric objects, but also in corrugated cylinders and hemicylindrical objects [16,17]. In [17], the authors report for the first time the existence of PNJ and WGM effects depending on forward/backward light illuminations in dielectric hemicylindrical particles. In fact, hemicylindrical aided nanojets show a relatively small influence of the WGM regime, when excited, in comparison to their cylindrical aided nanojet counterparts.

The vast majority of photonic nanojet studies employ either plane-wave source illumination [13] or Bessel-beam illumination [15] of dielectric micro-structures. The primary objective of this work is to investigate the generation of PNJs and WGMs supported by both smooth and corrugated dielectric micro-structures under point-source illumination. The work herein provides a demonstration of PNJ generation and WGM excitation under point-source illumination, which offers a more particle modelling scenario for microscopy where the sample is closer to the light source. The results presented in this work show that akin to plane-wave and Bessel-beam illuminations, point-source illumination not only generates PNJs but also exhibits WGMs, albeit with different electromagnetic dynamics and characteristics. In Ref. [18], the authors investigated the formation of photonic nanojets resulting from dielectric spheres illuminated by a focused beam. It is shown that the dimension of the jet beam waist is less sensitive when compared with plane-wave illumination. In addition, the authors in Ref. [19] investigated photonic nanojet formation due to a focused near-field diffraction.

Corrugation of the sidewall surface naturally occurs as a result of the imperfect nature of fabrication; however, in our work we consider a specific type of corrugation which possesses an azimuthal symmetry. There are many examples of photonic micro-structures with such geometrical properties. They include differently profilated fibers, see (Ref. [31]), or fibers with hexagonal cross section, see (Ref. [32]), which can be obtained by various fabrication technologies. Another example is represented by the hexagonally shaped high-index nanowires, as shown in [33,34]. In Ref. [35], in order to enhance the imaging resolution by WGMs, the authors proposed possible approaches based on an electric dipole formed by two incoherent point sources located near the micro-cylinders. Their analyses are based on Mie theory in which some dominant modes are selected for the investigation while ignoring the lower contributing near-field evanescent waves. In addition, in Ref. [35], the corrugated micro-cylinders have not been investigated. In contrast, in addition to the investigation of the latter point, the current work directly solves the governing electromagnetic wave equation to the electromagnetic structure.

The main contribution of the current work can be highlighted by two points; the first is the application of an illumination source that imitates practical wave excitation. In point-source illumination, the propagation directions of the wave fronts touching the surface of the micro-cylinder are not parallel to each other, as oppose to traditional plane-wave illumination. However, the second is the consideration of corrugation of the sidewall surface that occurs due to the imperfect nature of fabrication. In both cases, the generation of PNJ and WGMs are investigated. It is worthy to note that the point-source approximation is relevant to many physical situations. One of them is based on using, for example, photoexcited dye molecules. The molecules will re-radiate as point-like sources 
because their size is much smaller than the wavelength. The molecules can be deposited directly on the cylinder surface or located at some distance from it inside some liquids. One can certainly use other small light sources, such as semiconductor quantum dots.

In this work, the spectral element method (SEM) is used for the computation of scattered and transmitted fields. In fact, this method has been recently applied to electromagnetic scattering and/or radiation problems due to its accuracy and the relatively low computational requirements [36-41]. By way of example, the authors in Ref. [39] compared the computational load and relative error among SEM, finite element method and finite difference method in several one- and two-dimensional problems and reported exceptional accuracy in the case of SEM with significantly less computational cost.

The remainder of this paper is structured as follows: in Section 2, the computational modelling domain using the SEM is defined. Section 3, presents the results of point-source illumination for both PNJs and WGMs in the case of dielectric cylinders of constant radii, while Section 4 presents the results for corrugated cylinders. Conclusions are drawn in Section 5.

\section{Definition of the Computational Problem}

The computational domain consists of a dielectric cylinder (whose radius is $R$ and refractive index is $n$ ) existing in a medium whose refractive index is unity. As the domain is unbounded, the perfectly matched layer (PML) whose mathematical formulation is introduced in [38] is used for domain truncation. Figure 1 illustrates the schematic of the photonic structure in which $d$ represents the distance from the source to the cylinder's closest point, and $\theta=\arctan (R /(R+d))$ stands for the angle between propagation direction and the horizontal $x$-axis. In the background of Figure 1 , the electric field variation that represents the waves generated by point-source illumination is plotted. Here, the source (Green's function) is governed by the Helmholtz equation:

$$
\nabla^{2} E^{i n c}+k^{2} E^{i n c}=-\delta(\vec{r})
$$

in which $E^{i n c}$ denotes the electric field which is incident onto the cylinder, $k$ is the wave number, and $\delta(\vec{r})$ is the Dirac function. Since we are solving a two-dimensional problem the fields are independent of the $\mathrm{z}$ coordinate. Therefore, the line source turns to be a point source and the direction of propagation is perpendicular to the z-axis. The exact solution to Equation (1) can be expressed in terms of the zero-order Hankel function of the second kind: $E^{i n c}(\vec{r})=(j / 4) H_{0}^{(2)}(k|\vec{r}|)$. In Figure 2, the real part of the source field is plotted along the $x$-axis. The field decays rapidly near the source but as it propagates further away, it decays by $\approx 1 / \sqrt{r}$. In this type of illumination, the propagation directions of the waves fronts touching the surface of the cylinder are not parallel to each, which is quite different form plane-wave illumination. As $d$ approaches $\infty$ the modelling is reduced to that of plane-wave illumination.

In addition to regular cylinders defined by a constant radius $R$, we considered another type of cylinders whose surface is corrugated with a profile defined by the following equation:

$$
R p h i=R_{0}\left[1+\frac{\beta}{R_{0}} \sin \left(m_{1} \phi\right) \sin \left(m_{2} \phi\right)\right]
$$

where $R_{0}$ is the average radius, $\beta / R_{0}$ is the ratio of deformation of boundary to the average radius, $m_{1}$ and $m_{2}$ are the two periods of the variations of the corrugated boundary with $\phi$ being the azimuth angle. Please note that when either $m_{1}=0$ or $m_{2}=0$, Equation (2) is reduced to the cylinder with constant radius, $R_{0}$. 


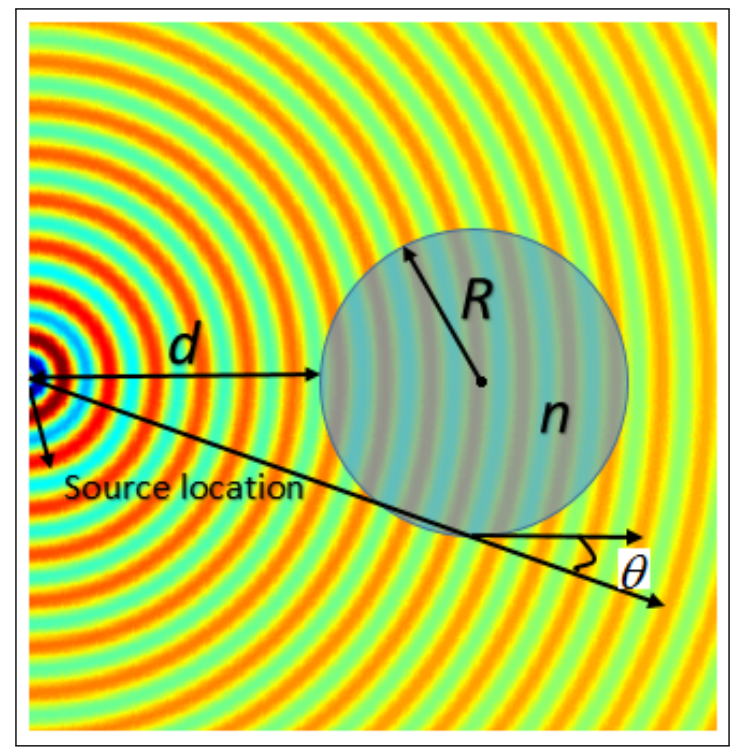

Figure 1. Schematic representation of the photonic structure illuminated by a point-source.

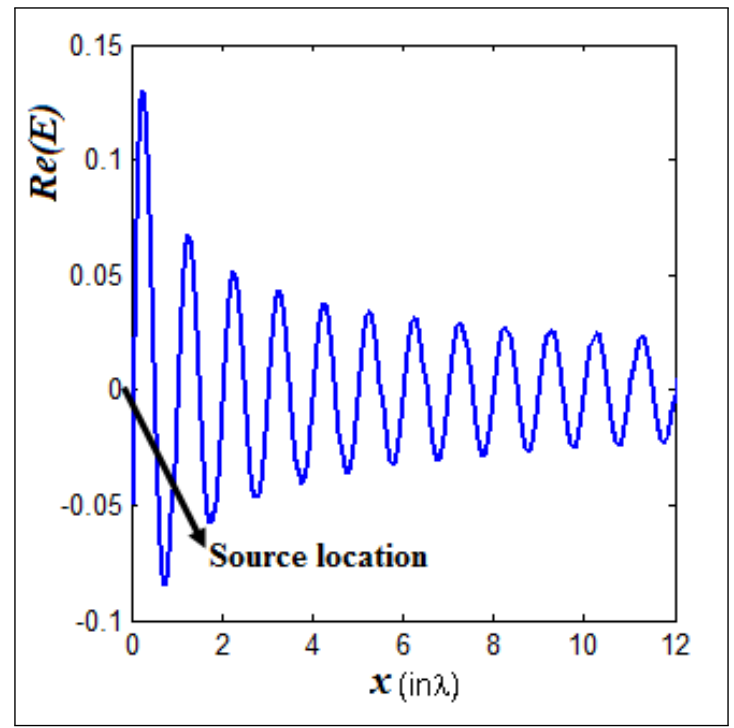

Figure 2. Real part of the electric field generated by a point-source. (x-axis is in terms of wavelength $\lambda$ ).

The following frequency-domain Helmholtz equation must be satisfied inside the computational domain (except the PML):

$$
\nabla^{2} E^{s c a}+(n k)^{2} E^{s c a}=k^{2}\left(1-n^{2}\right) E^{i n c}
$$

where $E^{s c a}$ stands for the scattered field. Since $n=1$ is assumed around the cylinder, Equation (3) reduces to the source-free homogenous Helmholtz equation.

Discretizing the electromagnetic domain by SEM into elements requires more care than in the typical cases of finite difference or finite element method. This is because irregular domains can be discretized by only deformed quadrilateral elements. A special type of meshing grid is deployed in the SEM to solve the field inside the computational domain. Gauss-Legendre-Lobatto (GLL) grids are used in the current work. To achieve the best accuracy offered by the SEM, Ref. [38] provides the optimum values of the attenuation factor associated with the number of nodes in GLLs. For the SEM formulation and SEM meshing, the reader may refer to $[12,16]$, respectively, for further details. 


\section{Illumination of Cylinders of Constant Radii}

To investigate PNJ generation, the point source is placed at a relatively close distance from the dielectric cylinder whose radius and refractive index are receptively $3 \lambda$ and 1.5, where $\lambda$ is the wavelength. In this work, the dimensions of the domain including the cylinder are normalized with respect to the incident wavelength. The photonic nanojets resulted from the point-source illumination are shown in Figure 3 when the source is placed at (a) $d=15 \lambda,(\mathrm{b}) d=10 \lambda,(\mathrm{c}) d=5 \lambda$, and (d) $d=3 \lambda$. Please note that the associate values of $\theta=9.5^{\circ}, 13^{\circ}, 20.6^{\circ}$, and $26.6^{\circ}$, respectively. Those values of $d$ are chosen as they represent critical transition and noticeable change in the output between having a perfect plane wave and a wave generated by a point source. However, the $\mathrm{R}$ and $\mathrm{n}$ are chosen to obtain a typical nanojet at a typical refractive index. Although the distance between the source and the cylinder's surface is provided, the calculated the values of $\theta$ are useful since they represent a measure of deviation from the plane-wave illumination case. Although one of the targets of this study is to investigate the effect of distance, and hence, the direction of propagating field as it deviates from plane-wave characteristics, we would like to mention for clarity that the field magnitudes as it touches the left edge of the cylinders were adjusted as $(d,|E|)=(3 \lambda, 0.06),(5 \lambda, 0.04),(10 \lambda, 0.03),(15 \lambda, 0.02)$.

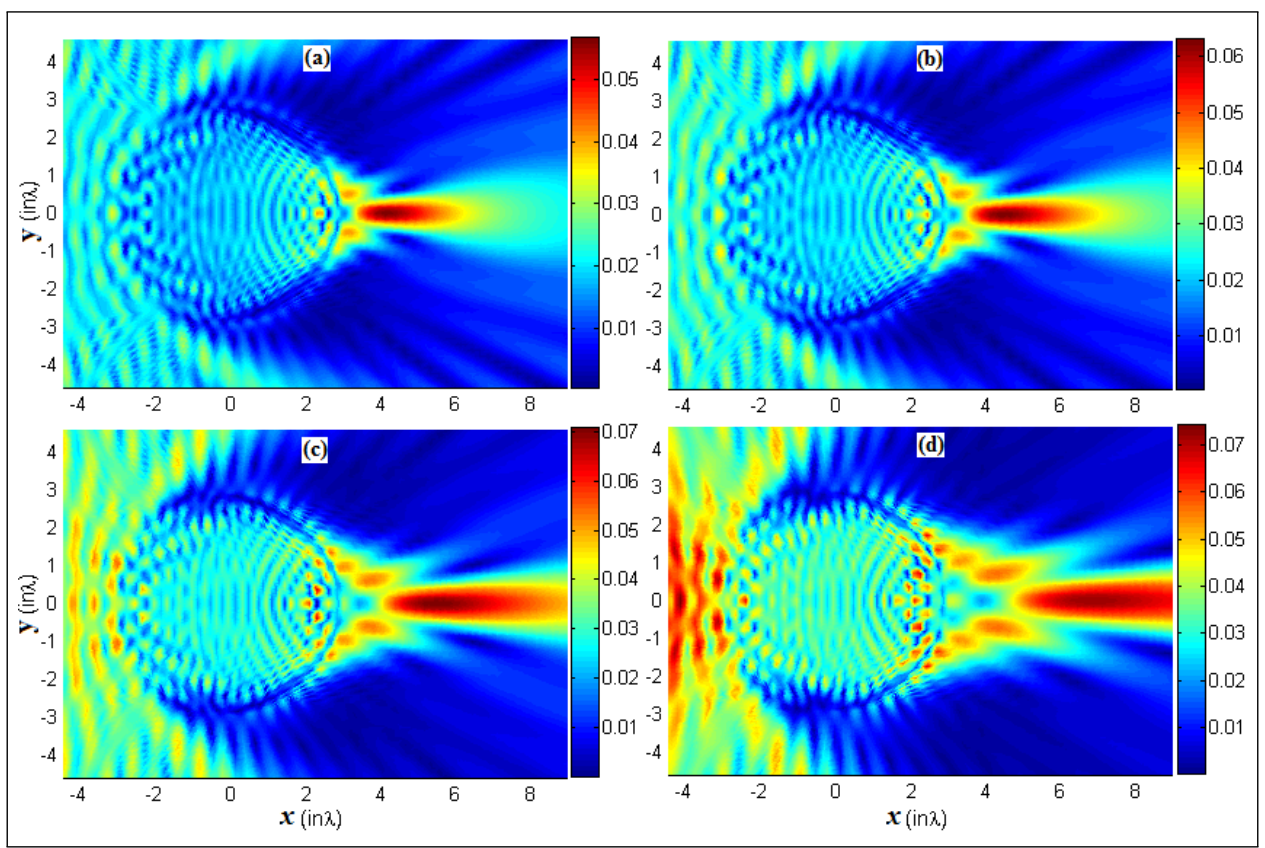

Figure 3. Illumination by a point-source placed at different positions from a cylinder whose $(R, n)=(3 \lambda, 1.5):(\mathbf{a}) d=15 \lambda,(\mathbf{b}) d=10 \lambda,(\mathbf{c}) d=5 \lambda$, and $(\mathbf{d}) d=3 \lambda$. (The colormap denotes the total field magnitude).

It can be clearly seen from the figure that as the source gets closer to the micro-cylinder, the photonic jet become longer in the $x$ direction and is excited further away from the cylinder's surface. This behavior is quite interesting, i.e., despite the fact that the incident wavelength, radius and refractive index of the cylinder, are fixed, still the characteristics of the nanojets can be altered by another parameter, namely the location of the point-source.

As stated in the introduction section, two illumination scenarios were so far applied in the literature; the first is the plane-wave illumination, and the other is Bessel-beam illumination. Under both scenarios, WGMs are captured. It is worthy to check whether WGMs still exist under other types of illumination. To check for the existence of WGMs, the following case $(R=3.06267 \lambda, n=1.95)$ is considered. The values of $R$ and $n$ were picked up from [15] at which WGM appears. Figure 4 shows the field magnitudes resulting from illumination by a point-source placed at two different positions: (a) $d=10 \lambda$, and (b) $d=5 \lambda$, and the associated field magnitudes along $\mathrm{x}$-axis are shown in (c) and (d), 
for (a) and (b), respectively. WGMs show stable behavior against the variation of the source position and this is contrary to photonic nanojet generation case. In other words, WGMs condition depends only on the refractive index $n$, radius of the cylinder $R$ and incident wavelength $\lambda$. To attract the readers' attention, we would like to note that WGMs exist for different point source locations, but the mentioned two cases are chosen for illustration purpose only.
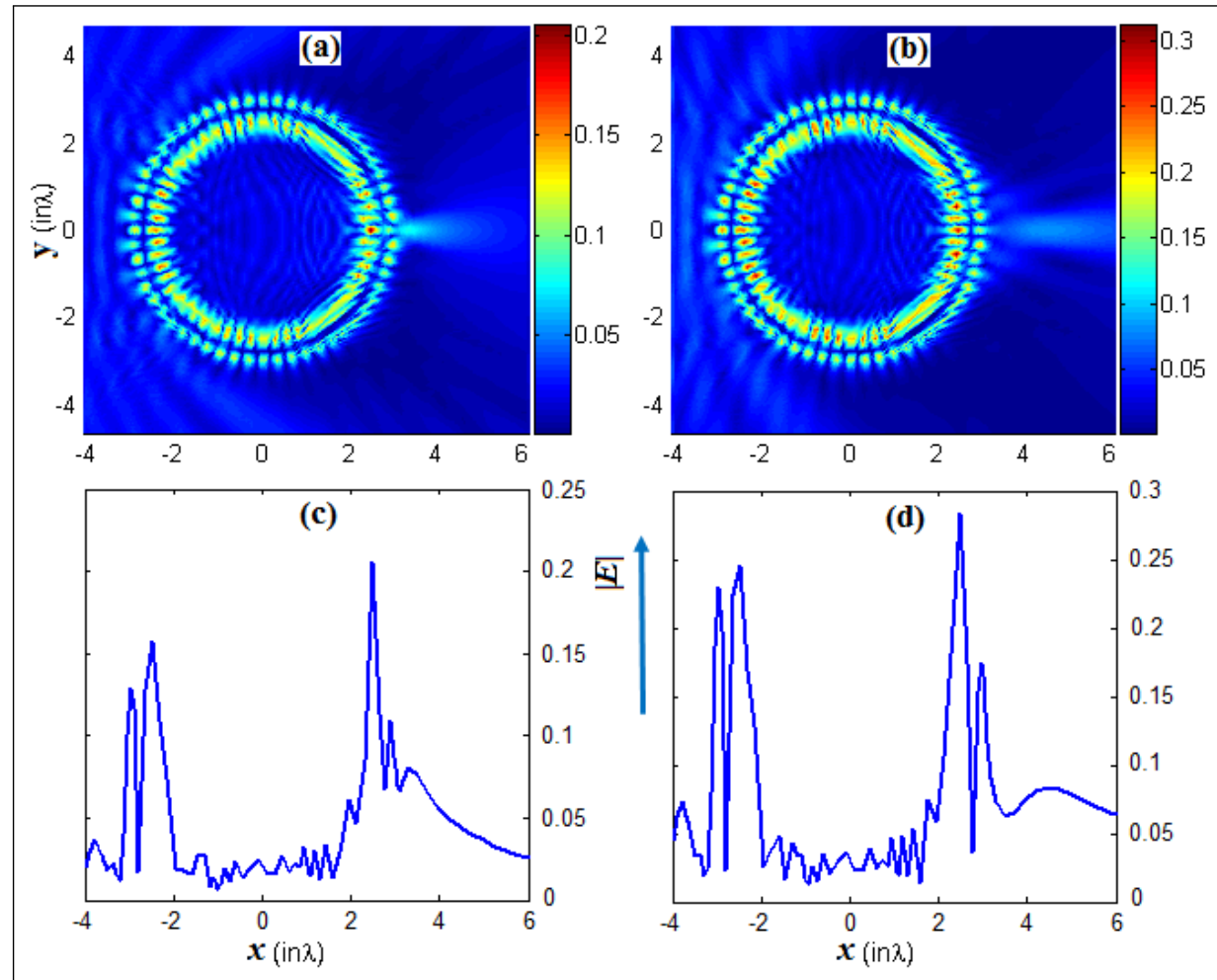

Figure 4. Illumination by a point-source placed at two positions: (a) $d=10 \lambda$, (b) $d=5 \lambda$, when $R=3.06267 \lambda, n=1.95$. The associated field magnitudes along $\mathrm{x}$-axis are shown in (c) and (d), respectively. (The colormap denotes the total field magnitude).

Here it is worthy to point out that SEM, as mentioned earlier, is highly dominating the finite difference or finite element methods in terms computational accuracy. In other words, one should not expect the total field distribution to be angularly symmetrical as in other studies where Mie theory is employed, and selective dominant modes are usually plotted. The reader may refer to $[13,15,16]$ for more information.

\section{Illumination of Corrugated Cylinders}

Numerical simulations for the point-source illumination of corrugated micro-cylinders are performed in this section. The simulation results indicate persistent photonic nanojet generation as with the smooth micro-cylinder case reported in the previous section. In Figure 5, at an average radius of $3 \lambda$ with a corrugation factor of $\beta$ and at constant refractive index of 1.4 , the magnitude of the total field is presented for (a) $d=15 \lambda, \beta=0.1$, (b) $d=15 \lambda, \beta=0.18$, (c) $d=5 \lambda, \beta=0.1$,(b) $d=5 \lambda$, $\beta=0.18$. Here, the two periods of the variations of the corrugated boundary $m_{1}$ and $m_{2}$ are set to 16 and 4, respectively. The corrugation depth in (a) and (c) is kept the same (whereas it is increased in (b) and (c)) to realize the effect of the illuminating source location. In the same figure, part (e) and part (f) are included as a reference, i.e., in order to realize the effect of corrugation, the total field is plotted at $n=1.4, R=3 \lambda$ for smooth cylinder at a distance of $d=15 \lambda$ in (e) and $d=5 \lambda$ in (f). As can be clearly observed, when the source gets closer to the cylinder, the PNJ is generated further away while being accompanied with stronger field localization at difference places near the cylinder's surface as 
a result of the corrugation profile and depth. The latter point is similar in the smooth cylinder case; however, the corrugation geometry leads to strong nearby field localization. In general, simulation results indicate that similar to the point-source illumination of smooth micro-cylinders, the corrugated micro-cylinders can sustain PNJs. Similarly, the point-source location acts as a degree of freedom for controlling the characteristics of the PNJ beyond the incident wavelength $\lambda$, average radius $R_{0}$, $m_{1}, m_{2}, \beta$ and refractive index $n$ of the corrugated micro-cylinder. In addition, the parameters of the corrugated micro-cylinder surface are then fixed with a refractive index $n=1.452$, a nominal average radius $R_{0}=3 \lambda, \beta=0.178$ and the two periods of the variations of the corrugated boundary $m_{1}$ and $m_{2}$ are set to 16 and 4 , respectively.

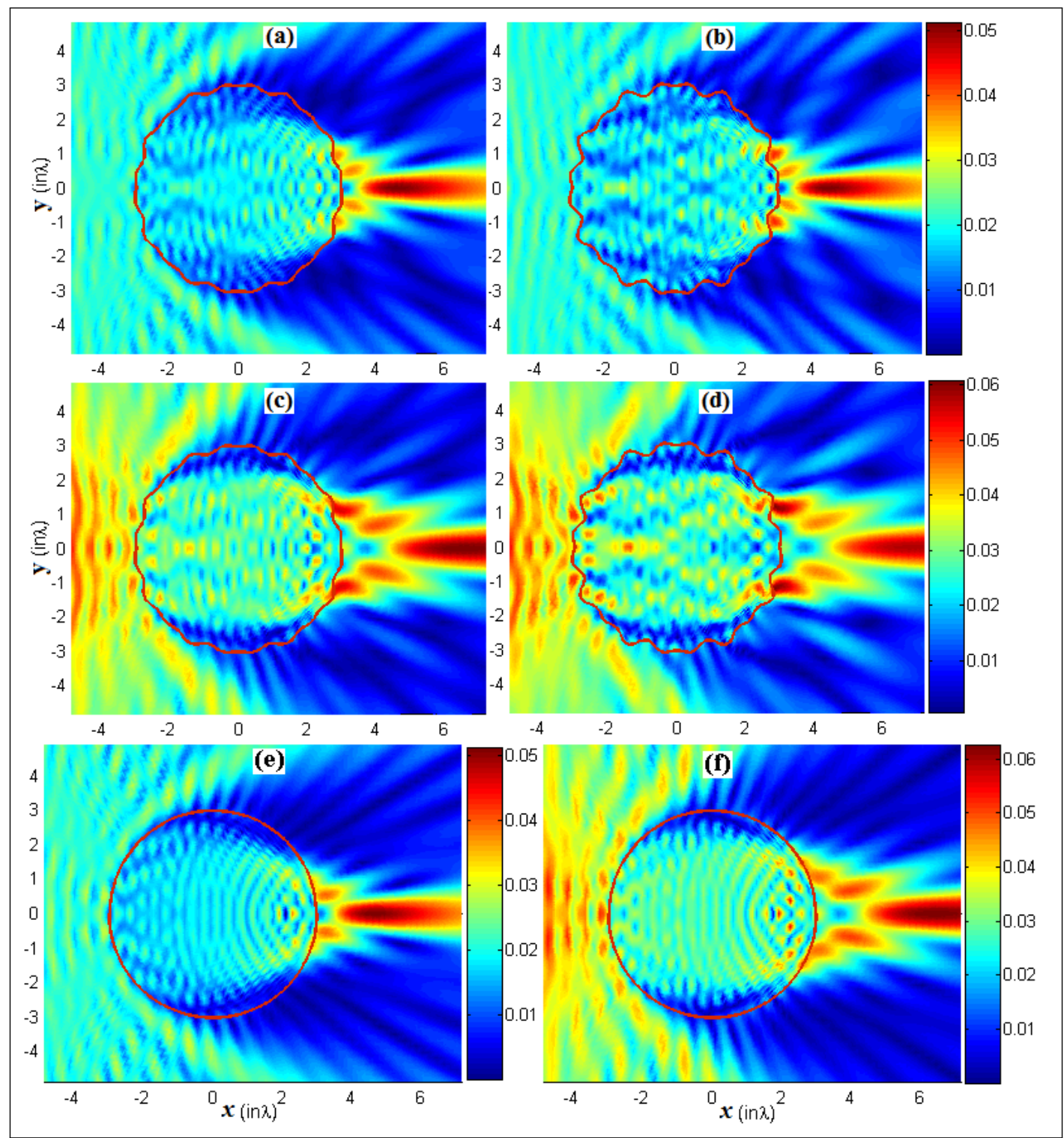

Figure 5. Total field inside and outside a dielectric corrugated cylinder whose $(R, n)=(3 \lambda, 1.4)$ when illuminated by a point-source placed at different positions (a) $d=15 \lambda, \beta=0.1$, (b) $d=15 \lambda, \beta=0.18$, (c) $d=5 \lambda, \beta=0.1$, (d) $d=5 \lambda, \beta=0.18$, (e) $d=15 \lambda, \beta=0$, (f) $d=5 \lambda, \beta=0$. (The colormap denotes the total field magnitude).

To investigate the excitation of WGMs from point-source illumination of corrugated micro-cylinders the source location is placed further away in an effort to emulate a plane-wave source. Here, we performed many simulations searching for the values of $\mathrm{n}$ that results in having 
WGMs and found to be 1.452 at the selected depth of corrugation. Simulation results shown in Figure 6 when the source is closely placed at $d=9 \lambda$, still indicate clearly that the excitation of WGMs is possible. However, as the point-source location is moved closer to the corrugated micro-cylinder the WGMs deteriorate into nanojets as depicted in Figure 7a,b, where the point-source location was set at $d=7 \lambda$, and $d=5 \lambda$, respectively. In contrast to the smooth micro-cylinder case of the previous section, which showed little dependence for the WGM stability on the distance $d$, this is not the case for the corrugated micro-cylinder, where the coherence of the WGMs deteriorate as the point-source is moved closer to the micro-cylinder surface. This is not all surprising as nanojets were reported for corrugated cylinders under normally incident plane-wave illumination [16].

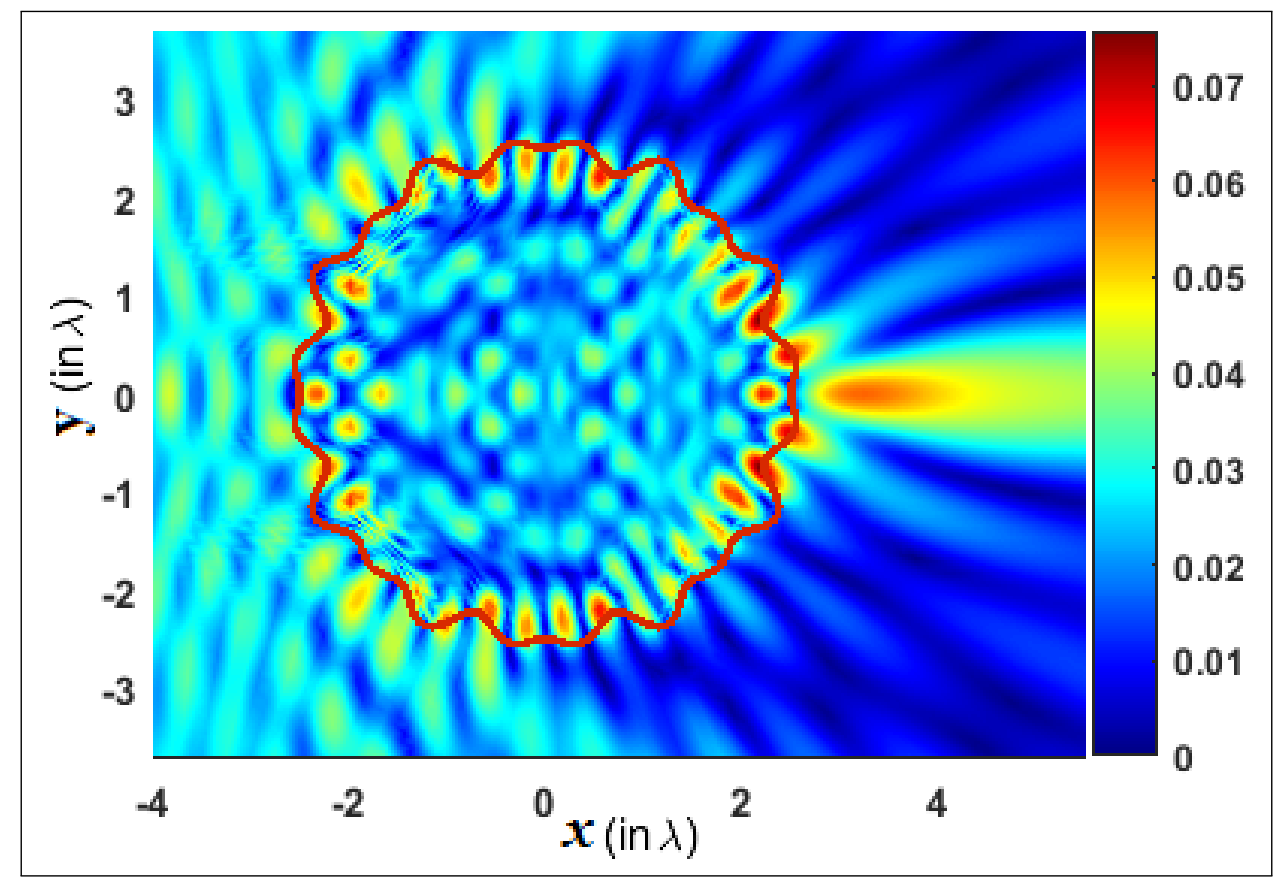

Figure 6. Illumination by a point-source placed at $9 \lambda, n=1.452,\left(m_{1}, m_{2}\right)=(16,4)$, and $\beta=0.178$. (The colormap denotes the total field magnitude).

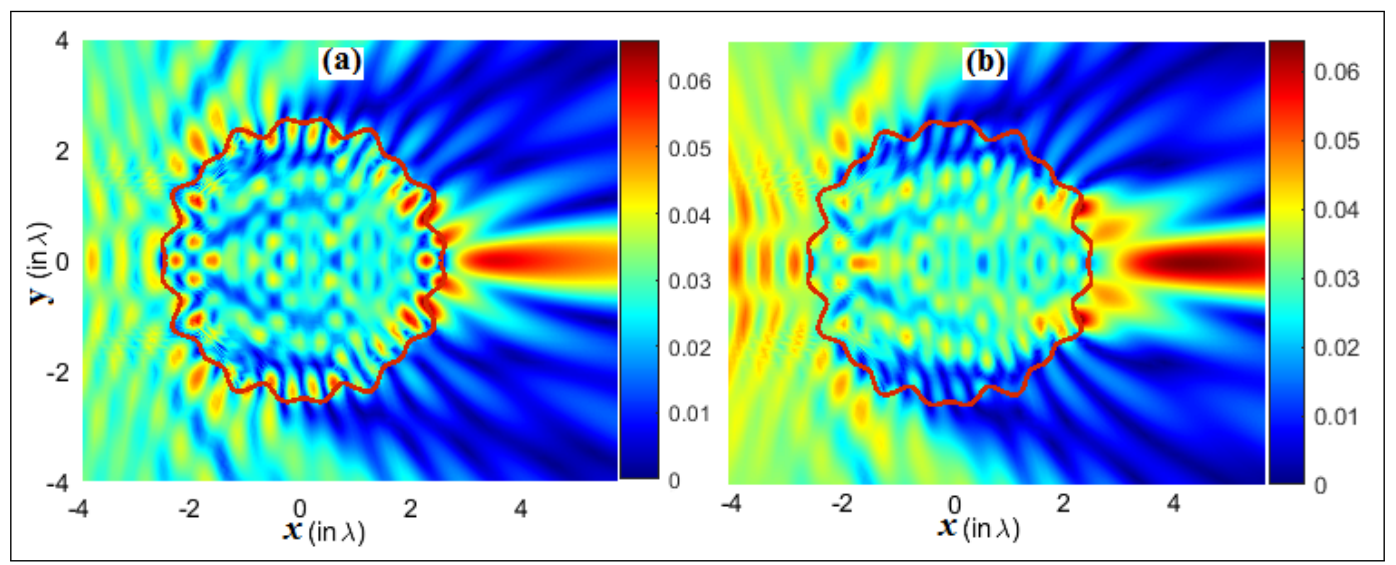

Figure 7. Illumination by a point-source for a cylinder with $n=1.452,\left(m_{1}, m_{2}\right)=(16,4)$, and $\beta=0.178$ placed at: (a) $d=7 \lambda$, and (b) $d=5 \lambda$. (The colormap denotes the total field magnitude).

\section{Conclusions}

The generation of PNJs and the excitation of WGMs supported by both smooth and corrugated dielectric micro-cylinders under point-source illumination were numerically investigated. Although the incident wave fronts onto the micro-cylinders were not parallel to each other, PNJs and 
stable WGMs were observed. It has been shown that the location of the point-source acts as an additional degree of freedom for controlling the characteristics of the generated PNJs for both smooth and corrugated dielectric micro-cylinders. Furthermore, the location of the point-source had little effect on the stability of the excited WGMs for smooth micro-cylinders. However, this was not the case for the corrugated micro-cylinders where the coherence of the WGMs deteriorated as the point-source was brought closer to the corrugated micro-cylinder surface.

Author Contributions: Conceptualization, I.M. and H.K.; methodology, I.M. \& H.K.; software, I.M.; validation, A.S.K. \& S.A.A.; formal analysis, T.A.; investigation, I.M. \& T.A.; resources, A.V.M. \& H.K.; writing-original draft preparation, I.M. \& T.A.; writing-review and editing, A.S.K. \& S.A.A.; supervision H.K. \& A.V.M. All authors have read and agreed to the published version of the manuscript.

Funding: This research received no external funding.

Conflicts of Interest: The authors declare no conflict of interest.

\section{References}

1. Benincasa, D.S.; Barber, P.W.; Zhang, J.-Z.; Hsieh, W.-F.; Chang, R.K. Spatial distribution of the internal and near-field intensities of large cylindrical and spherical scatterers. Appl. Opt. 1987, 26, 1348-1356. [CrossRef] [PubMed]

2. Luk'yanchuk, B.S.; Paniagua-Domínguez, R.; Minin, I.; Minin, O.; Wang, Z. Refractive index less than two: Photonic nanojets yesterday, today and tomorrow [Invited]. Opt. Mater. Express 2017, 7, 1820-1847. [CrossRef]

3. Geints, Y.E.; Zemlyanov, A.A.; Panina, E.K. Photonic jets from resonantly excited transparent dielectric microspheres. J. Opt. Soc. Am. B 2012, 29, 758-762. [CrossRef]

4. Chen, Z.; Taflove, A.; Backman, V. Photonic nanojet enhancement of backscattering of light by nanoparticles: A potential novel visible-light ultramicroscopy technique. Opt. Express 2004, 12, 1214-1220. [CrossRef]

5. Heifetz, A.; Huang, K.; Sahakian, A.V.; Li, X.; Taflove, A.; Backman, V. Experimental confirmation of backscattering enhancement induced by a photonic jet. Appl. Phys. Lett. 2006, 89, 221118. [CrossRef]

6. Kim, M.-S.; Scharf, T.; Mühlig, S.; Rockstuhl, C.; Herzig, H.P. Engineering photonic nanojets. Opt. Express 2011, 19, 10206-10220. [CrossRef]

7. Wang, Z.; Guo, W.; Li, L.; Luk'yanchuk, B.; Khan, A.; Liu, Z.; Chen, Z.; Hong, M. Optical virtual imaging at $50 \mathrm{~nm}$ lateral resolution with a white-light nanoscope. Nat. Commun. 2011, 2, 218. [CrossRef]

8. Dantham, V.R.; Bisht, P.B.; Namboodiri, C.K.R. Enhancement of Raman scattering by two orders of magnitude using photonic nanojet of a microsphere. J. Appl. Phys. 2011, 109, 103103. [CrossRef]

9. Kong, S.-C.; Sahakian, A.; Taflove, A.; Backman, V. Photonic nanojet-enabled optical data storage. Opt. Express 2008, 16, 13713-13719. [CrossRef]

10. Heifetz, A.; Kong, S.-C.; Sahakian, A.V.; Taflove, A.; Backman, V. Photonic nanojets. J. Comput. Theor. Nanosci. 2009, 6, 1979-1992. [CrossRef]

11. Wang, H.; Zhang, J.; Wu, X.; Shen, D. On-resonance photonic nanojets for nanoparticle trapping. Opt. Express 2019, 27,8 .

12. Mahariq, I.; Kurt, H.; Tarman, H.I.; Kuzuoglu, M. Photonic nanojet analysis by spectral element method. IEEE Photonics J. 2014, 6, 1-14. [CrossRef]

13. Mahariq, I.; Kurt, H. On and off optical resonance dynamics of dielectric micro-cylinders under plane wave illumination. J. Opt. Soc. Am. B 2015, 32, 1022-1030. [CrossRef]

14. Yang, J.; Twardowski, P.; Gerard, P.; Duo, Y.; Fontaine, J.; Lecler, S. Ultra-narrow photonic nanojets through a glass cuboid embedded in a dielectric cylinder. Opt. Express 2018, 26, 4. [CrossRef] [PubMed]

15. Mahariq, I.; Kurt, H. Strong field enhancement of resonance modes in dielectric microcylinders. J. Opt. Soc. Am. B 2016, 33, 656-662. [CrossRef]

16. Mahariq, I.; Astratov, V.; Kurt, H. Persistence of photonic nanojet formation under the deformation of circular boundary. J. Opt. Soc. Am. B 2016, 33, 535-542. [CrossRef]

17. Mahariq, I.; Giden, I.H.; Kurt, H.; Minin, O.V.; Minin, I.V. Strong electromagnetic field localization near the surface of hemicylindrical particles. Opt. Quant. Electron. 2018, 50, 423. [CrossRef] 
18. Dong, A.; Su, C. Analysis of a photonic nanojet assuming a focused incident beam instead of a plane wave. J. Opt. 2014, 16, 125001. [CrossRef]

19. Salhi, M.; Evans, P.G. Photonic Nanojet as a Result of a Focused Near-Field Diffraction. J. Opt. Soc. Am. B 2019, 36, 1031. [CrossRef]

20. Mahariq, I.; Eti, N.; Kurt, H. Engineering Photonic Nanojet Generation; Computational Electromagnetics International Workshop (CEM): Izmir, Turkey, 2015; pp. 1-4.

21. Minin, I.V.; Liu, C.-Y.; Geints, Y.E.; Minin, O.V. Recent Advances in Integrated Photonic Jet-Based Photonics. Photonics 2020, 7, 41. [CrossRef]

22. Minin, I.V.; Minin, O.V.; Geints, Y.E. Localized EM and photonic jets from non-spherical and non-symmetrical dielectric mesoscale objects: Brief review. Annalen der Physik 2015, 527, 491-497. [CrossRef]

23. Bonakdar, A.; Rezaei, M.; Brown, R.L.; Fathipour, V.; Dexheimer, E.; Jang, S.J.; Mohseni, H. Deep-UV microsphere projection lithography. Opt. Lett. 2015, 40, 2537-2540. [CrossRef] [PubMed]

24. Bonakdar, A.; Rezaei, M.; Dexheimer, E.; Mohseni, H. High-throughput realization of an infrared selective absorber/emitter by DUV microsphere projection lithography. Nanotechnology 2015, 27, 3. [CrossRef] [PubMed]

25. Biccari, F.; Hamilton, T.; Ristori, A.; Sanguinetti, S.; Bietti, S.; Gurioli, M.; Mohseni, H. Quantum dots luminescence collection enhancement and nanoscopy by dielectric microspheres. Particle Particle Syst. Charact. 2020, 37, 1900431. [CrossRef]

26. Darafsheh, A.; Bollinger, D. Systematic study of the characteristics of the photonic nanojets formed by dielectric microcylinders. Opt. Commun. 2017, 402, 270-275. [CrossRef]

27. Darafsheh, A. Influence of the background medium on imaging performance of microsphere-assisted super-resolution microscopy. Opt. Lett. 2017, 42, 735-738. [CrossRef]

28. Darafsheh, A.; Guardiola, C.; Palovcak, A.; Finlay, J.C.; Cárabe, A. Optical super-resolution imaging by high-index microspheres embedded in elastomers. Opt. Lett. 2015, 40, 5-8. [CrossRef]

29. Gérard, D.; Devilez, A.; Aouani, H.; Stout, B.; Bonod, N.; Wenger, J.; Popov, E.; Rigneault, H. Efficient excitation and collection of single-molecule fluorescence close to a dielectric microsphere. J. Opt. Soc. Am. B 2009, 26, 1473-1478. [CrossRef]

30. Devilez, A.; Bonod, N.; Wenger, J.; Gérard, D.; Stout, B.; Rigneault, H.; Popov, E. Three-dimensional subwavelength confinement of light with dielectric microspheres. Opt. Express 2009, 17, 2089-2094. [CrossRef]

31. Chongchang, Y.; Cheng, Q.; Wen, Z.; Yue, W.; Huaping, W. The design and manufacture of profiled multi-channeled hollow polyester fibers. Fibers Polym. 2009, 10, 657-661.

32. Podrazký, O.; Kašík, I.; Peterka, P.; Aubrecht, J.; Cajzl, J.; Proboštová, J.; Matějec, V. Preparation of optical fibers with non-circular cross section for fiber lasers and amplifiers. Proc. SPIE 2015, 9450, 94501A.

33. Gong, S.-H.; Ko, S.-M.; Jang, M.-H.; Cho, Y.-H. Giant Rabi splitting of whispering gallery polaritons in GaN/InGaN core-shell wire. Nano Lett. 2015, 15, 4517-4524. [CrossRef] [PubMed]

34. Xu, C.; Dai, J.; Zhu, G.; Zhu, G.; Lin, Y.; Li, J.; Shi, Z. Whisperinggallery mode lasing in ZnO microcavities. Laser Photonics Rev. 2014, 8, 469-494. [CrossRef]

35. Zhou, S.; Deng, Y.; Zhou, W.; Yu, M.; Urbach, H.P.; Wu, Y. Effects of Whispering Gallery Mode in Microsphere Super-Resolution Imaging. Appl. Phys. B 2017, 123, 9. [CrossRef]

36. Mehdizadeh, O.Z.; Paraschivoiu, M. Investigation of a twodimensional spectral element method for Helmholtz's equation. J. Comput. Phys. 2003, 189, 111-129. [CrossRef]

37. Lee, J.; Xiao, T.; Liu, Q.H. A 3-D spectral-element method using mixed-order curl conforming vector basis functions for electromagnetic fields. IEEE Trans. Microw. Theory Tech. 2006, 54, 4141-4148. [CrossRef]

38. Mahariq, I.; Kuzuoğlu, M.; Tarman, H.I. On the attenuation of perfectly matched layer in electromagnetic scattering problems with spectral element method. Appl. Comput. Electromagn. Soc. J. 2014, 29, 701-710.

39. Mahariq, I.; Kurt, H.; Kuzuoğlu, M. Questioning degree of accuracy offered by the spectral element method in computational electromagnetics. Appl. Comput. Electromagn. Soc. J. 2015, 30, 698-705. 
40. Mahariq, I.; Erciyas, A. A spectral element method for the solution of magnetostatic fields. Turk. J. Elec. Eng. Comp. Sci. 2017, 25, 2922-2932. [CrossRef]

41. Mahariq, I. On the application of the spectral element method in electromagnetic problems involving domain decomposition. Turk. J. Elec. Eng. Comp. Sci. 2007, 25, 1059-1069. [CrossRef]

(C) 2020 by the authors. Licensee MDPI, Basel, Switzerland. This article is an open access article distributed under the terms and conditions of the Creative Commons Attribution (CC BY) license (http:/ / creativecommons.org/licenses/by/4.0/). 